\title{
The Use of Psychophysiological Measures for Designing Adaptive Learning Systems
}

\author{
Stamos T. Karamouzis \\ Loyola University New Orleans, \\ Computer Information Science Department \\ 6363 St. Charles Ave,, New Orleans, LA 70185, USA \\ stamos@loyno.edu
}

\begin{abstract}
When we trace the history of mind and learning theories we clearly see a transitioning course that traverses Cartesianism, Behaviorism, and finally Functionalism. Current advances in computer scanning technologies reinforce the view that learning should also be examined under the prism of braincentered materialist theories. Adaptive learning systems are instructional technologies that try to minimize the mismatch between learner needs and the learning environment. Currently, they try to elicit the learner needs with performance measures but they ignore learner differences at the brain level. This paper offers a shift of viewpoint in thinking about future adaptive learning systems. If we want education to be precisely tailored to the needs of learners then instructional technologies must take advantage of known individual differences in brain processing. The paper offers the justification of such an approach, analyzes its implications, proposes an implementation model, reviews related work, and outlines future challenges.
\end{abstract}

\section{Introduction}

The history of computer-based learning systems can be traced back as far as the beginning of computing. Since the early computing days an effort was made to create learning systems that adapt their functionality based on the learner needs and capabilities thus giving birth to the class of adaptive learning systems. The most notable early work in adaptive learning systems was done by B. F. Skinner in the 1960s. Skinner and his colleagues developed the concept of programmed learning where a teaching machine engages a learner in a task, and uses a comparison of the learner's performance to a model of how the task should be performed in order to provide the learner with advice to successfully complete the task $[27,28]$.

Through the years, evolutionary forces resulted in the development of learning systems that represent a leap beyond the earlier Skinnerian work in terms of their capacity to detect learner abilities and deficiencies as well as to provide insightful

Please use the following format when citing this chapter:

Karamouzis, Stamos, 2006, in IFIP International Federation for Information Processing, Volume 204, Artificial Intelligence Applications and Innovations, eds. Maglogiannis, I., Karpouzis, K., Bramer, M., (Boston: Springer), pp. 417-424 
remediation. In the traditional mode of operation adaptive learning systems maintain the domain knowledge that needs to be conveyed to the learner (domain module), the learner's existing knowledge (learner's module), and the pedagogical methods that can be used in order to convey the domain knowledge (methods module). Through the interaction of these modules the learning systems are able to make judgments about the student's knowledge, skills, and progress. The methods module tailors the learning experience to the student's needs, automatically, without the intervention of a human instructor [1, 3].

Today's adaptive learning systems are capable of modifying any individual student's learning experience as a function of information obtained through their performance on situated tasks or assessments [12]. Situated tasks can be any dynamic learning challenge that requires a measurable response on the part of the learner. These can include problem sets, learning task challenges, a Socratic dialogue, or experiences in simulated environments. In a nutshell, the adaptation is done based only on performance measurements.

When we trace the history of mind and learning theories we clearly see a transitioning course that traverses Cartesianism, behaviorism, and finally functionalism [13]. Current advances in computer scanning technologies reinforce the view that learning should also be examined under the prism of brain-centered materialist theories.

Nowadays, advances in psychophysiological techniques, such as brain scanning, make it possible to identify differences in human brain processing that correspond to differences in learning styles and capabilities. The knowledge of such differences is valuable in every educational setting because teaching methods that may work well for a large majority of learners may be counterproductive when used with learners that their brain processing deviates from the majority. Additionally the same psychophysiological techniques may reveal, in real time, the learner's cognitive state.

Therefore, with this article, I propose the design of adaptive learning systems that incorporate the use of psychophysiological measurements, in addition to performance measurements, in modifying the student's learning experience. I assert that such systems will be more effective and I hope to stimulate new ideas and research in this direction.

In particular, I give a brief overview of the relevant psychophysiological techniques, I explain how such techniques may benefit learning theories, I propose a new implementation model for adaptive learning systems, and I outline future challenges.

\section{Psychophysiology}

Psychophysiology research and practice deals with the interactions between the mind and body by recording how the body is functioning and relating the functions recorded to behavior. The field is based on the premise that changes in the body's functioning cause changes in behavior and vice versa [9]. Psychophysiological recording techniques are generally non-invasive. That is, they record from the body's surface and nothing goes into the person being recorded. 
Nowadays, the filed of Psychophysiology is employing a number of techniques that help elicit data about the function and structure of the brain. They can be divided into those that provide functional information and those that provide structural information about the brain [5].

\subsection{Structural Measures}

The magnetic resonance imaging (MRI), magnetic resonance spectroscopy (MRS), and diffusion tensor imaging (DTI) are methods used to measure brain structure and chemistry. For example, magnetic resonance imaging (MRI) can be used to measure gross size or volume differences in brain regions while magnetic resonance spectroscopy (MRS) can be used to measure the concentration of cerebral metabolites that have been related to neuronal loss or damage. Thus MRS can provide additional insight as to why an MRI-based measure of a brain structure may be smaller. Finally, diffusion tensor imaging (DTI) allows for measures of the regularity and myelination of fiber tracts and provides a more precise measure of myelination of fibers than traditional MRI measures of white matter volume. All three of these structural imaging methods can be correlated with behavior, but none involves simultaneous collection of behavior or the capability of measuring brain changes associated with trial-by-trial behavior.

\subsection{Functional Measures}

In contrast, Electroencephalography (EEG), event related potentials (ERP), single photon emission computed tomography (SPECT), positron emission tomography (PET), functional magnetic resonance imaging (fMRI), near infrared spectroscopy/optimal imaging (NIRS), and magnetoencephalography (MEG) are classified as functional imaging methods because they measure changes in brain activity associated with simultaneous changes in behavior.

In particular, Electroencephalography (EEG) is a method for capturing and measuring brain waves and as such provides evidence of how the brain functions over time. It is commonly used for detecting and observing certain conditions, such as seizures, by observing changes in the normal pattern of the brain's electrical activity $[8,30]$. The output from an EEG is recorded as a graph of brainwaves on a time scale and simply reveals rough brainwave frequency and amplitude. Measuring Event Related Potentials (ERP) involves correlating the EEG brainwave response with a stimulus (event) and averaging the result of dozens to thousands of stimulus expositions together to get a clear picture of what electrical activity takes place upon presentation of that specific stimulus [2]

A Single Photon Emission Computed Tomography (SPECT) scan is a type of nuclear imaging test that shows how blood flows to tissues and organs. This is done by ejecting the body with a radioactive chemical that emits gamma rays and can be detected by a scanner. SPECT scans are relatively inexpensive and readily available. [14]

Positron emission tomography (PET) is a technique that produces a threedimensional image or map of functional processes in the body based on the detection 
of radiation from the emission of positrons. This is achieved by injecting into the body a short-lived radioactive tracer isotope that decays by emitting the detected positrons [4]. This technique differs from a SPECT scan in that the chemical is being absorbed by surrounding tissues rather than staying in the blood stream, therefore the images are not limited to areas where blood flows.

Functional magnetic resonance imaging ( $\mathrm{MMRI}$ ) is a technique that exposes the brain with a high static magnetic field and a small alternating radiofrequency field. Thus is capable of visualizing changes in chemical composition of brain areas or changes in the flow of fluids that occur over time periods of seconds to minutes. It can be used to find out what the brain is doing when subjects perform specific tasks or are exposed to specific stimuli [6]. There are three advantages to fMRI scaning over PET scanning 1) It is not invasive since the signal does not require injections of radioactive isotopes, 2 ) the total scan time required can be very short, i.e., on the order of 1.5 to $2.0 \mathrm{~min}$ per run, and 3) it is capable of fine resolutions in the order of $1.5 \times 1.5 \mathrm{~mm}$ or even less.

Near infrared spectroscopy (NIRS) offers a safe, non-invasive means of monitoring cerebral function without the use of radioisotopes or other contrast agents. NIRS systems measure the oxygenated blood flow through the brain by shining light in the near infrared (NIR) range of the spectrum through the scalp [31].

Magnetoencephalography (MEG) is noninvasive technology for functional brain mapping that is based on the measurement of intercellular currents of the neurons in the brain, spontaneously or to a given stimulus [19]. MEG combines many of the advantages of PET and $\mathrm{AMRI}$ scanning but MEG's temporal resolution of $1 \mathrm{~ms}$ is far superior while having an equivalent spatial resolution.

\section{Psychophysiology \& Learning}

Functional imaging techniques are capable of measuring subtle task-induced changes in signals from the brain and as such they can be of particular interest in formulating learning theories and constructing learning environments. In general, they can be useful in the filed of learning for three reasons. First they can shed light into understanding how learning occurs. Second they can identify differences in learning styles and capabilities. Third they can monitor in real time and reveal an individual's cognitive state.

\subsection{Understanding Learning}

Through the years a number of learning theories have been developed usually aligned along the philosophical theories of the time. Nowadays, the advances in brain scanning technologies allow us to develop a better understanding and insight of how learning occurs and thus piece-by-piece either validate or refute existing theories. For example imaging studies using $\mathrm{AMRI}$ of the prefrontal cortex helped identify how humans focus attention on the task at hand while ignoring distractions $[7,16]$. Those studies are significant because, contrary to current learning theories, 
prove that attention control is achieved by amplifying task-relevant information, rather than by inhibiting distracting stimuli.

\subsection{Identifying Differences}

Over the past two decades researchers from various labs were able to use structural and functional brain imaging techniques in identifying learner differences such as learners with dyslexia and attention deficit disorder $[10,20,25,26]$.

For example, Richards and Berninger $[21,25]$ used functional magnetic resonance spectroscopy and fMRI to show that there are chemical differences between the brains of learners with dyslexia and those of other learners. Their work is based on monitoring metabolic activity of the brain. When the brain is at work, it uses energy. One by-product of energy use in the brain is lactate. By measuring where lactate is being produced, they were able to see which part of the brain was active.

Identifying learner abilities is crucial in an educational setting in order to choose the pedagogical methodology that matches best the learner's abilities. For example in teaching reading, pedagogical methods based on intensive or systematic drill in phonemic awareness or phonetic decoding strategies may actually be harmful to dyslexic learners. Such teaching might simply emphasize reliance on mental strategies that are as likely to diminish reading ability for dyslexics, instead of improve it.

\subsection{Revealing Cognitive States}

Since the early 90's researches proposed the use of Psychophysiology for eliciting cognitive states. In 1995, Pope, Bogart, and Bartolome developed a system that used EEG in airplane simulators to monitor when pilots are engaged and when they're disengaged during a typical flight. The pilot's level of engagement was then used by a system that adapted the airplane's level of automation. When pilots became disengaged, the airplane became less automated and the additional work required pulled the pilots back into the process of flying the aircraft $[17,18]$.

Expanding upon the work of Pope and his colleagues, St. John, Kobus, Morrison, and Schmorrow have described a new DARPA program aimed at developing systems that can detect an individual's cognitive state and then manipulate task parameters to overcome four primary "bottlenecks" in cognitive performance: attention, executive functioning, sensory input, and working memory [29].

Although Pope's and St. John's work aimed at developing adaptive automation systems that enhance an operator's effectiveness the same principles and finding are applicable in learning systems that can adapt to better fit learner needs. In fact, one of Pope's striking findings was the similarity between the brain waves of disengaged pilots and the brain waves of learners with Attention Deficit Disorder. 


\section{A Model for Adaptive Learning Systems}

The power that psychophysiological measures give us in understanding how learning occurs, in identifying individual learning differences, and in revealing cognitive states can be invaluable for every educational setting.

In particular, for designing an adaptive learning system I propose that such measures should be integral part of the learner's module in order to transform the domain and the instructional methods modules. In all of the existing systems the learner's module is exclusively comprised of the learner's motives and knowledge as it is being assessed by performance measures. The incorporation of psychophysiological findings can greatly improve the accuracy of the learner's module. This can be done at two distinct collection levels.

First, a battery of psychophysiological tests should be administered to assess the student's learning style and abilities. This will create an individualized profile, diagnostic in style, which gives answers to the questions: Is the learnet an auditory personality? visual? tactile/kinesthetic? dyslexic? etc. This psychophysiological profile should be incorporated into the learner's module along with the information from the performance measurements thus resulting in an augmented learner's module. At the second collection level real-time information about the learner's cognitive state should be collected. The psychophysiological monitoring should match specific learning materials and tasks with constructs such as effort, arousal, attention, and workload.

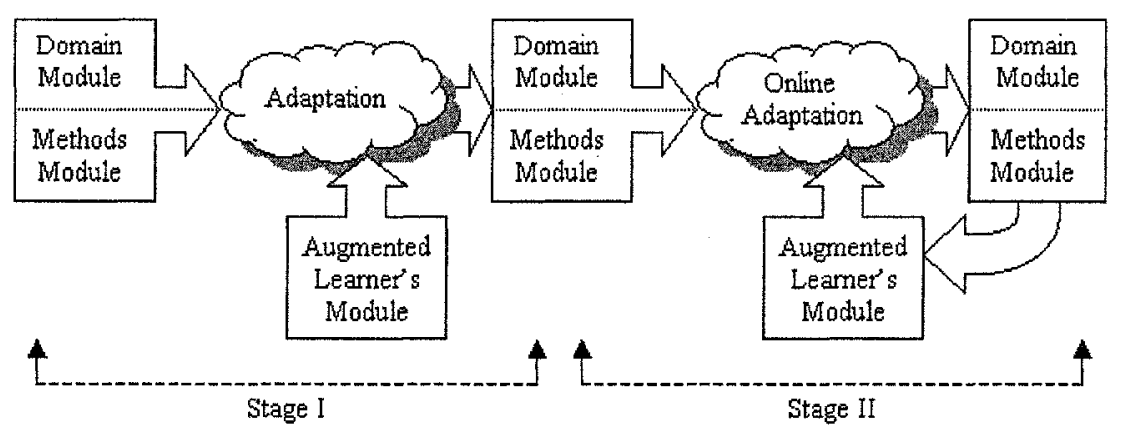

Fig. 1. Model for an Adaptive Learning System

In traditional adaptive learning systems Artificial Intelligence (AI) techniques have been instrumental in providing the adaptation of the domain and methods modules. Based on this model AI techniques should also be applied for interpreting the psychophysiological findings. To this day only limited work has been done in this direction $[22,23]$.

The existence of those two levels of collecting psychophysiological measurements leads us into a two-stage model for adaptive learning systems (figure 1). At Stage I, the initialization stage, the domain knowledge and the set of available pedagogical methodologies get customized based on the initial learner's module that includes the psychophysiological profile from the first collection level. Stage II, the real-time 
stage, further adapts the domain and methods modules in a dynamic way as the second level collection occurs. Such adaptation may include reprioritizing or rescheduling tasks, changing the level of difficulty, or switching between verbal and spatial information formats.

\section{Discussion}

The notion of using psychophysiology for explaining learning processes was first introduced by Tom Mulholland in 1974 but since no work has been achieved [15]. Nowadays, the impressive advances in psychophysiological techniques progressed our abilities to understand how learning occurs, identify individual learning differences, and reveal cognitive states. Despite the isolated successes many significant challenges must be overcome if the proposed model for adaptive learning systems is to move out of the laboratory.

First of all, more experimentation needs to be done. Today, the work done in augmented cognition does not have learning in mind [24]. Future experimentation needs to focus on learning, learning differences, and cognitive states as they specifically relate to learning tasks. Second, researchers in this area need to establish the validity of the approach through systematic controlled tests and replication.

Additionally, serious effort needs to be invested in order to achieve remote psychophysiological monitoring [11]. Only when we eliminate the wiring and physical contact between machine and humans we'll be able to truly develop applications outside the laboratory.

Also, numerous other issues such as determining the frequency of adaptive changes, adaptive algorithms, types of interfaces, improving the signal-to-noise ratio of psychophysiological measures, extracting useful signals from within dynamic environments, etc. need to be addressed. Nonetheless, the proposed model poses as a starting point in an exciting direction that holds enormous promise for the future of adaptive learning systems.

\section{References}

1. Alberink, M. \& Veenstra, M. A framework for adaptable hypermedia authoring. TI/RS/2001/067 Enschede, Telematica Instituut (2001)

2. Brandeis, D., Lehmann, D.: Event-related potentials of the brain and cognitive processes: approaches and applications. Neuropsychologia 24 (1986) 151-68

3. Brusilovsky, P., Peylo, P.: Adaptive and Intelligent Web-based Educational Systems. International Journal of Artificial Intelligence in Education 13 (2003) 156-169

4. Carson, R., Herscovitch, P., Daube-Witherspoon, M.: Quantitative Functional Brain Imaging With Positron Emission Tomography. Elsevier (1998)

5. Casey, B.J., de Haan, M.: New methods in developmental science. Developmental Science 5 (2002) 265-267

6. Cohen, M.S., Bookheimer, S.Y.: Localization of brain function using magnetic resonance imaging. Techniques in Neuroscience 17 (1994) 268-277

7. Egner T., Hirsch, J.: Cognitive control mechanisms resolve conflict through cortical amplification of task-relevant information. Nature Neuroscience 8 (2005) $1784-1790$

8. Emerson, R. G., Pedley, T.A.: Electroencephalography and evoked potentials. In: Neurology in Clinical Practice. Vol. 1, Butterworth-Heinemann (2000) 473-485 
9. Frank, Y., Pavlakis, S.G.: Brain imaging in neurobehavioral disorders. Pediatric Neurology 25 (2001) 278-287

10. Giedd, J.N., Blumenthal, J., Molloy, E., Castellanos, F.X.: Brain Imaging of Attention Deficit/Hyperactivity Disorder. Annals of the New York Academy of Sciences 931 (2001) $33-49$

11. Jemison, M., Mandavilli, R., Wang, S., Morgan-Reynolds, R.: Using Remote Psychophysiological Monitoring for Human Performance T\&E. ITEA Journal March/April (2004) 15-16

12. Jones, M., Winne, P.H.: Foundations and Frontiers of Adaptive Learning Enviranments. Springer Verlag (1992)

13. Lyons, W.: Matters of the Mind. Edinburgh University Press (2001)

14. Masdeu, J.C., Brass, L.M., Holman, B.L., et al.: Special review: Brain single photon emission tomography. Neurology 44 (1994) 1970-1977

15. Mulholland, T.B.: Training visual attention. Academic Therapy 10 (1974) 5-17

16. Nieuwenhuis, S., Yeung, N.: Neural mechanisms of attention and control: losing our inhibitions? Nature Neuroscience 8 (2005) 1631 - 1633

17. Pope, A.T., Bogart, E.H., Bartolome, D.: Biocybernetic system evaluates indices of operator engagement. Biological Psychology, 40 (1995) 187-196

18. Prinzel, L.J., Pope, A.T., Freeman, F.G., Scerbo, M.W., Mikulka P.J.: Empirical Analysis of EEG and ERPs for Psychophysiological Adaptive Task Allocation. NASA/TM-2001211016, National Aeronautics and Space Administration (2001)

19. Ribary, U., Mogilner, A., Joliot, M., Volkman, J., Rusinek, H., Llinas, R.: The use of magnetoencephalography (MEG) and magnetic resonance imaging (MRI) to localize normal and pathological human brain function. Radiology (1992) 185-199

20. Richards, T.L.: Functional Magnetic Resonance Imaging and Spectroscopic Imaging of the Brain: Application of FMRI and fMRS to Reading Disabilities and Education. Learning Disabilities Quarterly 24 (2001) 189 - 203

21. Richards, T.L., Berninger, V.W., Aylward, E.H., et al.: Reproducibility of proton MR spectroscopic imaging (PEPSI): comparison of dyslexic and normal-reading children and effects of treatment on brain lactate levels during language tasks. American Journal of Neuroradiology 23 (2002) 1678-85

22.Russell, C., Wilson, G.: Feature Saliency Analysis for Operator State Estimation. Proceedings of the $11^{\text {th }}$ International Conference on Human-Computer Interaction (2005)

23. Russell, C., Wilson, G.: Comparing Classifiers for Real Time Estimation of Cognitive Workload. Proceedings of the $11^{\text {th }}$ International Conference on Human-Computer Interaction (2005)

24. Scerbo, M.W.: Biocybernetic Systems: Information Processing Challenges that Lie Ahead. Proceedings of the $11^{\text {th }}$ International Conference on Human-Computer Interaction (2005)

25. Serafini, S., Steury, K., Richards, T.D, Abbott, R., Berninger, V.: Comparison of AMRI and PEPSI during language processing in children. Magn Reson Med 45 (2001) 217-225

26. Shaywitz, S.: Overcoming Dyslexia. Random House (2005)

27. Skinner, B.F.: Why we need teaching machines. Harvard Educational Review 31 (1961) $377-98$

28. Skinner, B.F., Holland, J.G.: The use of teaching machines in college instruction (Parts IIIV). In A. A. Lumsdaine \& R. Glaser (Eds.), Teaching machines and programmed learning: A source book. Washington, DC: Department of Audio-Visual Instruction, National Education Association (1960) 159-172

29. St. John, M., Kobus, D.A., Morrison, J.G., Schmorrow, D.: Overview of the DARPA Augmented Cognition technical integration experiment. International Journal of HumanComputer Interaction (2004) 131-149.

30. Tyner, F.S., Knott, J.R., Meyer, W.B.: Electroencephalography. New York: Raven Press; (1983)

31. Villringer, A., Chance, B.: Non-invasive optical spectroscopy and imaging of human brain function. Trends in Neurosciences 20 (1997) 435-442. 